Rev. Elev. Méd. vét. Pays trop., 1978, 31 (1) : 79-83.

\title{
Un exemple d'utilisation de la méthode actuarielle : l'évaluation des taux de mortalité au sein d'un troupeau ovin de race Timahdit
}

\author{
par J. CABARET $\left({ }^{*}\right)$
}

\begin{abstract}
RÉSUMẼ
La méthode actuarielle pour les anniversaires a été utilisée sur un troupeau de race Timahdit. Son avantage principal par rapport aux méthodes classiques est l'utilisation des animaux durant leur présence dans le troupeau, même si elle est courte. Elle est particulièrement intéressante pour l'étude des taux de mortalité sur des périodes longues. Elle est simple et rapide ; sa précision est bonne et permet d'obtenir des taux de mortalité homogènes au cours des diverses périodes. Cette méthode ne s'applique qu'à des populations de faible effectif et parfaitement définies. Ces inconvénients sont compensés par le fait qu'elle utilise durant leur temps de présence les animaux perdus ou vendus. Elle semble donc intéressante pour l'appréciation des taux de mortalité des troupeaux à gestion moderne.
\end{abstract}

La connaissance des taux de mortalité est un moyen pour apprécier la conduite d'un troupeau. Diverses méthodes existent pour évaluer ces taux. Elles diffèrent par 2 aspects : le mode de la prise d'information, la population prise en compte. L'information peut être prélevée à date fixe (tous les mois par exemple) ou au contraire aux dates d'anniversaires des animaux. La première éventualité a l'avantage de la simplicité ; par contre il existe une incertitude. En effet, une mort peut avoir lieu peu après ou juste un peu avant le passage de l'enquêteur. La méthode des anniversaires permet de dater exactement une mortalité ; elle présente l'inconvénient de nécessiter une information tenue au jour le jour.

Deux techniques quant à la population utilisée. Supposons que l'on désire connaître le taux de mortalité à 1 an pour des ovins. La méthode directe consiste à utiliser les indices suivants :

- Nombre de décès au bout d'1 an/nombre

(*) Institut Agronomique et Vétérinaire Hassan II Bloc Vétérinaire, B. P. 704, Rabat, Agdal, Maroc. de présents au début de la période (1 an) âgés de $0-1$ mois.

- Nombre de décès au bout d'un an/nombre moyen de présents au cours de la période ( $1 \mathrm{an}$ ) âgés de $0-1$ mois $\left(^{*}\right)$.

La première estimation se rapproche d'un quotient de mortalité, la seconde représente un taux de mortalité vrai. Ces estimations présentent un biais important lorsqu'un grand nombre d'animaux est exclu du troupeau pour des raisons diverses (vente en particulier). Ainsi, dans cet exemple, un animal vendu à 9 mois ne sera pas utilisé ; il en sera de même pour un animal né 3 mois après le début de l'enquête. L'intérêt de la méthode actuarielle est de faire rentrer dans les calculs ce type d'animal; à la notion de recul ( 1 an), on substitue celle d'histoire (présent pendant 9 mois dans le troupeau). On calculera donc les taux de survie des animaux présents, par exemple, pendant 3 mois,

(*) La moyenne est réalisée sur le nombre d'animaux âgés de 0-1 mois au début et à la fin de la période d'étude (un an). 
6 mois, 9 mois et 1 an. Le taux de survie à 1 an sera donc : (Survie 0-3 mois) $\times$ (Survie 3-6 mois) $\times$ Survie (6-9 mois) $\times$ Survie (9-12 mois). On peut se demander pourquoi on utilise les taux de survie. C'est que la formule des produits de probabilités concernant des événements successifs n'est valable que pour les échecs; or on mesure la mortalité, l'échec est donc la survie. On obtient le taux de mortalité à une période en prenant le complément à 1 du taux de survie.

On dispose donc, pour mesurer les taux de mortalité, de 4 possibilités :

- Méthode directe :

— information à date fixe,

- information aux anniversaires.

- Méthode actuarielle :

- information à date fixe,

- information aux anniversaires.

La méthode actuarielle pour les anniversaires est celle qui inclut le plus d'information, et par suite apporte la meilleure précision pour l'évaluation des taux de mortalité. C'est la raison pour laquelle nous l'avons choisie.

Elle est souvent employée en médecine humaine (1) et peut s'adapter facilement à l'étude des taux de mortalıté chez les animaux.

\section{MATÉRIEL}

Nous avons utilisé les carnets d'agnelage de la race Timahdit de la ferme d'application de l'Institut Agronomique et Vétérinaire Hassan II, située à Moghrane (Maroc).

\section{MÉTHODE}

II. 1. Nomenclature et intervalle de confiance des résultats.

A chaque période de temps considérée sont associées 7 valeurs (3) qui sont :

1) vivants au début de l'intervalle: $V_{k}$,

2) décédés dans l'intervalle: $D_{k, k+1}$,

3) vendus ou perdus dans l'intervalle:
$E V_{k, k+1}$ (il faut exclure les abattages d'urgence $(*)$,

4) groupe présent durant tout l'intervalle : $N_{k}$,

5) taux de mortalité pour la période : $\mathrm{M}_{\mathrm{k}, \mathrm{k}+1}(* *)$,

6) taux de survie pour la période: $S_{k, k+1}$,

7) taux de survie à la période $\mathrm{k}+1=\mathrm{S}_{\mathrm{k}+1}$.

On en déduit facilement le taux de mortalité à la période $\mathrm{k}+1$.

Les 4 dernières données sont calculées comme suit :

$$
\begin{aligned}
& \mathrm{N}_{\mathrm{k}}=\mathrm{V}_{\mathrm{k}}-\mathrm{EV}_{\mathrm{k}, \mathrm{k}+1} \quad(1,4) \\
& \mathrm{M}_{\mathrm{k}, \mathrm{k}+1}=\frac{\mathrm{D}_{\mathrm{k}, \mathrm{k}+1}}{\mathrm{~N}_{\mathrm{k}}} \\
& \mathrm{S}_{\mathrm{k}, \mathrm{k}+1}=1-\mathrm{M}_{\mathrm{k}, \mathrm{k}+1} \\
& \mathrm{~S}_{\mathrm{k}+1}=\mathrm{S}_{0-1} \times \mathrm{S}_{1-2} \ldots \mathrm{S}_{\mathrm{k}, \mathrm{k}+1}\left({ }^{* * *}\right) .
\end{aligned}
$$

La variance des divers taux de survie $S_{k}$ ne peut être calculée par les méthodes classiques concernant les pourcentages; on utilise la formule approximative de GREENWOOD (2) :

$$
\begin{array}{r}
\text { Var. } S_{k}=S_{k}^{2}\left[\frac{M_{0,1}}{N_{0}\left(1-M_{0,1}\right)}+\frac{M_{1,2}}{N_{1}\left(1-M_{1,2}\right)}+\right. \\
\left.+\cdots+\frac{M_{k-1, k}}{N_{k}\left(1-M_{k-1, k}\right)}\right] .
\end{array}
$$

Les taux de mortalité obtenus ont un intervalle de confiance de $\pm 2 \sqrt{\text { Var. } S_{k}}$.

\section{II.2. Réalisation pratique}

a) Définir chaque intervalle de temps pour lequel on désire connaître le taux de mortalité.

b) Compter le nombre d'animaux présents dans l'intervalle total d'étude : $(\mathrm{N})$.

c) Relever les dates de naissance, de mort des animaux morts et remplir le tableau suivant :

(*) Les abattages d'urgence correspondent à un décès, même s'ils donnent lieu à une vente.

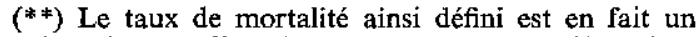
quotient de mortalité. Nous conservons cette dénomination, bien qu'inexacte, celle-ci étant toujours employée dans ce genre de problème.

$\left(*^{* *}\right)$ Les taux de survie ne sont plus calculés dès que le $V_{k}$ correspondant est inférieur à 10 (1). En effet la variance devient élevée et l'évaluation de ces taux est sujette à une trop grande imprécision. 


\begin{tabular}{|l|c|c|c|}
\hline Date de naissance & Date de mort & $\begin{array}{c}\text { Durée en jours } \\
\text { qui sépare } \\
\text { ces deux dates }\end{array}$ & $\begin{array}{c}\text { Intervalle } \\
\text { auquel l'animal } \\
\text { appartient }\end{array}$ \\
\hline & &
\end{tabular}

Les valeurs obtenues dans la $3^{\text {e }}$ colonne permettent d'effectuer un intervalle à chaque animal. Supposons que l'on trouve 300 jours, on dira que cet animal appartient à l'intervalle 0-1 an.

d) Relever les dates de naissance, de vente des animaux vendus et remplir un tableau semblable au précédent.

e) Remplir la $1^{\text {re }}$ partie du tableau définitif.

\begin{tabular}{|c|c|c|c|}
\hline Intervalle & $\begin{array}{c}\text { Vivants } \\
V_{k}\end{array}$ & $\begin{array}{c}\text { Morts } \\
D_{k, k+1}\end{array}$ & $\begin{array}{c}\text { Vendus } \\
\text { EV } k, k+1\end{array}$ \\
\hline $0-1$ & $\mathrm{~N}$ & $\mathrm{a}$ & $\mathrm{b}$ \\
\hline $1-2$ & $\mathrm{~N}-\mathrm{a}-\mathrm{b}$ & $\mathrm{c}$ & $\mathrm{d}$ \\
\hline $2-3$ & $\mathrm{~N}-\mathrm{a}-\mathrm{b}-\mathrm{c}-\mathrm{d}$ & & \\
\hline
\end{tabular}

f) La seconde partie du tableau définitif $\left(N_{k}, M_{k, k+1}, S_{k, k+1}, S_{k+1}\right)$ est établie par le calcul, dans l'ordre énoncé plus haut.

\section{RÉSULTATS}

Les données disponibles concernant le troupeau ovin de la ferme de Moghrane sont traitées par la méthode actuarielle pour les anniversaires et les étapes du calcul sont représentées dans les tableaux I et II.

Le premier, qui concerne l'ensemble du troupeau en 1975, illustre l'éventualité où le nombre d'exclus vivants est important. Le second tableau présente le cas inverse.

TABL. $N^{\circ} I$ : Mortalité du troupeau (mâles et femelles) en 1975.

\begin{tabular}{|c|c|c|c|c|c|c|c|}
\hline $\begin{array}{l}\text { Intervalle } \\
\text { (en mois) }\end{array}$ & $v_{k}$ & $\mathrm{D}_{\mathrm{k}, \mathrm{k}}+1$ & $\mathrm{EV}_{\mathrm{k}, \mathrm{k}}+1$ & $\mathrm{~N}_{\mathrm{k}}$ & $M_{k}, k+1$ & $s_{k, k}+1$ & $\mathrm{~s}_{\mathrm{k}}+1$ \\
\hline $0-2$ & 101 & 4 & 0 & 101 & 3,96 & 96,04 & 96,04 \\
\hline $2-4$ & 97 & 4 & 0 & 97 & 4,12 & 95,88 & 91,12 \\
\hline $4-6$ & 93 & $I$ & 3 & 90 & 1,11 & 98,89 & 90,11 \\
\hline $6-8$ & 89 & 3 & 11 & 78 & 3,85 & 96,15 & 86,64 \\
\hline $8-10$ & 75 & 1 & 0 & 75 & 1,33 & 98,67 & 85,49 \\
\hline $10-12$ & 74 & 2 & 11 & 63 & 3,17 & 96,83 & 82,78 \\
\hline $12-14$ & 61 & 1 & 2 & 59 & 1,69 & 98,31 & 81,38 \\
\hline $14-17$ & 58 & 2 & 0 & 58 & 3,45 & 96,55 & 78,57 \\
\hline
\end{tabular}

TABL. $\mathrm{N}^{\circ} \mathrm{II}$ : Mortalité des femelles du troupeau du $28-4-74$ au 2-4-77.

\begin{tabular}{|c|c|c|c|c|c|c|c|}
\hline $\begin{array}{l}\text { Intervalle } \\
\text { (en mois) }\end{array}$ & $v_{k}$ & $D_{k, k}+1$ & $\mathrm{EV}_{\mathrm{k}, \mathrm{K}}+1$ & $\mathrm{~N}_{\mathrm{k}}$ & $M_{k, k}+1$ & $s_{k, k}+1$ & $S_{k}+1$ \\
\hline $0-1$ & 130 & 4 & 0 & 130 & 3,07 & 96,93 & 96,93 \\
\hline $1-3$ & 126 & 4 & 5 & 121 & 3,30 & 96,70 & 93,72 \\
\hline $3-6$ & 117 & 5 & 4 & 113 & 4,42 & 95,58 & 89,57 \\
\hline $6-12$ & 108 & 5 & 2 & 106 & 4,72 & $95,2 B$ & $B 5,34$ \\
\hline $12-18$ & 101 & 2 & 0 & 101 & 1,98 & 98,02 & 83,65 \\
\hline $18-24$ & 99 & 0 & 0 & 99 & - & - & 83,65 \\
\hline $24-30$ & 99 & 0 & 0 & 99 & - & - & 83,65 \\
\hline
\end{tabular}




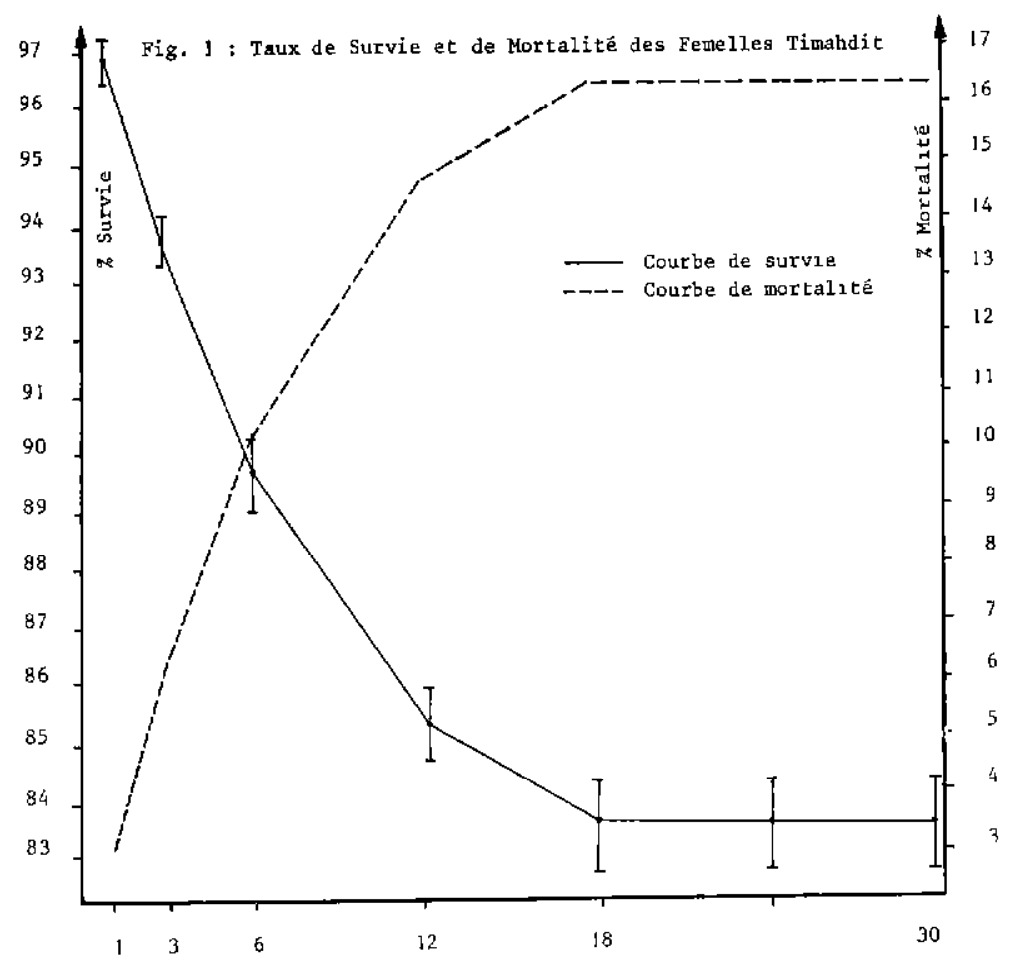

On notera que l'on passe facilement des taux de survie aux taux de mortalité, dont l'usage est plus répandu. Ainsi, pour le troupeau dans son ensemble en 1975, le taux de mortalité pour les 2 premiers mois est $100-96,04$, soit 3,96 .

La figure 1 représente les taux de survie (tabl. II) avec leurs intervalles de confiance ainsi que les taux de mortalité.

\section{DISCUSSION}

La méthode actuarielle par les anniversaires présente un intérêt différent suivant les âges considérés et les sexes. En effet, dans les premiers mois, il n'y a pas d'animaux commercialisés et la méthode n'apporte rien. L'intérêt est sensible pour le troupeau dans son entier en 1975, car beaucoup de mâles sont vendus ; ceci est d'autant plus vrai que l'âge des animaux est élevé.

Les taux de mortalité obtenus dans les derniers intervalles, par 2 méthodes, illustrent ces considérations.

\begin{tabular}{|c|c|c|}
\hline & \multicolumn{2}{|c|}{ Information aux anniversaires } \\
\cline { 2 - 3 } & $\begin{array}{c}\text { Tableau I } \\
\text { (dernier } \\
\text { intervalle) }\end{array}$ & $\begin{array}{c}\text { Tableau II } \\
\text { (dernier } \\
\text { intervalle) }\end{array}$ \\
\hline Méthode directe ... & $24,3 \pm 12,2 \%$ & $16,8 \pm 6,85$ \\
\hline Méthode actuarielle & $21,4 \pm 8,7 \%$ & $16,3 \pm 6,68$ \\
\hline
\end{tabular}

La méthode actuarielle est plus précise (intervalle de confiance plus petit) en particulier pour les données du tableau I ( 27 p. 100 de vendus). L'intérêt est plus faible pour le troupeau de femelles (tabl. II) où seulement 0,5 p. 100 des animaux sont vendus.

Elle est plus homogène que la méthode directe, car elle diminue l'incidence des ventes sur les résultats finals. La comparaison de deux troupeaux, de deux classes d'âge, bien que les opérations commerciales soient intervenues d'une façon différente, pourra être réalisée avec un maximum d'efficacité, car les variations dues aux seules ventes sont éliminées en partie. 
On utilise dans ce cas la formule suivante fondée sur l'écart réduit (3).

$$
\varepsilon=\frac{S_{k}-S_{k^{*}}}{\sqrt{\text { Var. } S_{k}+\text { Var. } S_{k^{*}}}}\left(^{*}\right) .
$$

La différence est significative à 5 p. 100 quand $\varepsilon$ est $\geqslant 1,96$. C'est le cas pour les périodes $6-12$ et suivantes par rapport à celle $0-1$ mois pour le troupeau de femelles. Cela veut dire que l'essentiel de la mortalité se produit entre 0 et 1 mois.

(*) On suppose que $S_{k}$ et $S_{k}$, suivent une distribution normale et cela n'est vrai que pour des effectifs importants $(\geqslant 30)$.
Nous n'aurions pu aboutir à cette conclusion en utilisant la méthode directe : aucune différence n'est significative.

\section{CONCLUSION}

La méthode actuarielle est plus précise que les méthodes directes; elle est aussi rapide. Elle nécessite toutefois certaines conditions : il faut que les divers taux de mortalité et que les effectifs qui servent à les calculer soient assez grands.

Elle est donc la méthode de choix lorsque l'on désire comparer les mortalités de divers lots à des fins expérimentales et ceci d'autant plus que le nombre d'animaux exclus (ou vendus) est élevé ( 10 p. 100 et plus).

\section{SUMMARY}

An example of the actuarial method utilization : estimate of death rate in Timahdit sheep herd

The actuarial method for birthdays is used on a flock of sheep of Timahdit breed. Its main advantage, compared to classical methods, is the utilization of animals during the time they belong to the flock, even if short. It is particularly interesting for the study of death rate over long periods. It is easy and quick; its accuracy is good and allows homogeneous death rates along the various periods.

This method can only be applied to small populations, perfectly knowned. This disavantages are balanced with the fact that it makes use of the animals during all their stay in the flock, until they are lost or sold. The method seems interesting for estimate of death rate in modern-managed flocks.

\section{RESUMEN}

Un ejemplo de utilización del método actuarial :

la evaluación de las tasas de mortalidad en un rebaño de ovejas de raza Timahdit

Se utilizó el método actuarial para los aniversarios en un rebaño de raza Timahdit. Su ventaja principal en relación con los metodos clásicos es la utilización de los anımales durante su presencıa en el rebaño más que sea corta. Es particularmente interesante para el estudio de las tasas de mortalidad durante largos periodos.

Es simple y rápida ; su precisión es buena y permite obtener tasas homogéneas de mortalidad durante los varios périodos.

Dicho método sólo se aplica en las poblaciones poco importantes y perfectamente definidas.

Utiliza durante su tiempo de presencia los animales perdidos o vendidos, lo que compensa estos inconvenientes.

Asi pues parece interesante para la apreciación de las tasas de mortalidad de los rebaños explotados de modo moderno.

\section{BIBLIOGRAPHIE}

1. FABIANI (J. N.), CARPENTIER (A.). La méthode actuarielle pour l'analyse statistique des résultats cliniques et expérimentaux. Nouv. Presse méd., 1977, $6(5): 357-361$.

2. GREENWOOD (M.). A report on the natural duration of cancer. Appendix I : the " Errors of Sampling 》 of the survivorship tables. London, Stationary Office,
1926 (Reports on Public Health and Medical subject no 35-Ap. I).

3. SCHWARTZ (D.), FLAMANT (R.), LELLOUCH (J.). L'essai thérapeutique chez l'homme. Paris, Flammarion (Médecine, Sciences), 1970, p. 211-237.

4. SCHWARTZ (D.), LELLOUCH (J.). Méthode de calcul des taux de survic. Bull. Cancer, 1959, 46 : 489495. 